\title{
Therapy of inflammatory bowel disease: A surgeon's perspective
}

\author{
ZANE COHEN MD FRCSC FACS
}

\begin{abstract}
Z COHEN. Therapy of inflammatory bowel disease: A surgeon's perspective. Can J Gastroenterol 1996;10(1): 43-47. Management of patients with inflammatory bowel disease (IBD) can be extremely challenging and difficult. IBD patients, however, must be well aware of all therapy options and must be involved in the decision-making process. Both medical and surgical therapies have their own resultant toxicity and morbidity which must be taken into consideration. Gastroenterologists and surgeons must come together in their thinking about IBD patients and continue to undertake trials of both medical and surgical therapies in order to determine the best management for any individual patient. Topics including controversies related to the pelvic pouch procedure, stricturoplasty in the management of Crohn's disease, assessment of trials using methotrexate and cyclosporine, and toxicity of medications and surgery are highlighted.
\end{abstract}

Key Words: Crohn's disease, Cyclosporine, Inflammatory bowel disease, Pelvic pouch, Stricturoplasty, Ulcerative colitis

\section{Traitement des maladies inflammatoires de l'intestin : point de vue du chirurgien}

RÉSUMÉ : Le traitement des patients atteints de maladies inflammatoires de l'intestin (MII) peut être un défi considérable. Or ces patients doivent toutefois être au courant de toutes les options thérapeutiques et participer aux prises de décision. Les traitements médicaux et chirurgicaux comportent tous deux des éléments de toxicité et de morbidité dont il faut tenir compte. Les gastro-entérologues et les chirurgiens doivent faire front lorsqu'il est question de MII et continuer leurs essais sur les traitements médicaux et chirurgicaux pour en arriver aux traitements les mieux adaptés aux patients sur le plan individuel. Les thèmes abordés ici sont, entre autres, les controverses entourant les interventions pour la construction de réservoirs iléaux, la stricturoplastie dans le traitement de la maladie de Crohn, l'évaluation des essais sur le méthotrexate et la cyclosporine, la toxicité des médicaments et la chirurgie.
$\mathrm{T}$ he options of surgical therapy for ulcerative colitis patients include total proctocolectomy and Brooke ileostomy, total proctocolectomy and continent ileostomy, subtotal colectomy and ileorectal anastomosis, as well as the pelvic pouch procedure. A stoma is required for the first two procedures and no stoma is required for subtotal colectomy, ileorectal anastomosis and pelvic pouch procedure. In our experience, as well as that of others, there are very few candi- dates that are now accepted for colectomy and ileorectal anastomosis alone. All the above procedures achieve continence except for conventional ileostomy, but the reoperation rates for all the procedures vary from $25 \%$ to $50 \%$. The reoperation rate for continent ileostomy is greater than $25 \%$ with colectomy and ileorectal anastomosis, and approximately 10 to $12 \%$ following the pelvic pouch proce-

Department of Surgery, University of Toronto, Toronto, Ontario

Correspondence and reprints: Dr Z Cohen, Chief of Surgery, Mount Sinai Hospital, 451-600 University Avenue, Toronto, Ontario M5G 1X5. Telephone 416-586-8346, fax 416-586-8644

This paper was presented at the Trends in Inflammatory Bowel Disease Therapy meeting, April 6 to 9, 1994, held in Victoria, British Columbia. This paper has also been published in Sutherland LR, et al, eds. Inflammatory Bowel Disease: Basic Research, Clinical Implications and Trends in Therapy. Boston, Dordrecht and London: Kluwer Academic Publishers, 1994 
dure. With all types of surgeries, there may be failures of therapy. As will be discussed, the morbidity following a pelvic pouch procedure is considerable and must be weighed against the benefits of performing a total proctocolectomy and Brooke ileostomy. However, surgery is curative for ulcerative colitis and avoidance of colectomy should not be the major outcome measure when one is assessing a patient for either medical or surgery therapy. The disease is completely removed with any form of total proctocolectomy and in most cases with the pelvic pouch procedure, although recently the transitional zone, as well as 1 to $2 \mathrm{~cm}$ of anal mucosa, may be left behind using a stapled technique for the pelvic pouch procedure. There have been no cancers reported in any of the pouches made for either the continent ileostomy or the pelvic pouch procedure. However, there have been two reported cases of cancer originating in the rectal cuff of patients who have had an incomplete mucosectomy where the indication for surgery was dysplasia (1).

Therefore, surgical options for patients with ulcerative colitis must be presented in light of the psychological and psychosocial problems that may occur when patients undergo a total proctocolectomy and Brooke ileostomy versus the technical difficulties, surgical complications and functional outcome that result from the pelvic pouch procedure.

In our experience since the mid-1970s, the procedure of choice for patients with ulcerative colitis has shifted from total proctocolectomy and Brooke ileostomy to continent ileostomy initially, and, since the early 1980s, to the pelvic pouch procedure almost exclusively. There are very few patients who are considered suitable candidates for continent ileostomy and only a limited number of patients who choose total proctocolectomy and Brooke ileostomy. Colectomy and ileorectal anastomosis in ulcerative colitis have become rare procedures in our experience.

\section{CONTROVERSIES RELATED TO THE PELVIC POUCH PROCEDURE}

The pelvic pouch procedure has become the operation of choice in our institution for patients undergoing surgery for ulcerative colitis. However, areas of controversy remain. These include pouch configuration, anal sphincter pressure changes, the original diagnosis and pouchitis. Although there have been numerous studies with the J, S, H, K and W pouches, I believe that pouch configuration is not of much significance. The key point is to have the ability to create more than one type of pouch in case the pouch configuration has to be changed to achieve more length to do a tensionfree ileoanal anastomosis. It is well known that resting anal pressure decreases following pelvic pouch surgery and never returns to normal, although this does not seem to influence the functional results to a great extent. The original diagnosis is also important in that patients undergoing pelvic pouch surgery for ulcerative colitis have a poorer outcome in general than those undergoing the same surgery for familial polyposis. Pouchitis is a clinical syndrome that usually manifests with fatigue, anemia, changes in stool consistency and frequency, and often an endoscopic appearance of acute or chronic inflammation. Only in our series has there been an association with stenosis at the outlet (2). Regarding age, it is well described that patients older than 50 years have a poorer result than younger patients (3).

Quality of life assessments are quite difficult. In an attempt to delineate whether pelvic pouch patients have a better outcome than those undergoing Kock pouch or conventional ileostomy, 36 patients who had had pelvic pouch procedure, 28 with a Kock pouch and 30 with conventional ileostomy were studied (4). These patients were chosen at random, but all had had their surgery at least one year previously and were in stable condition. Using both the time trade-off technique and the direct questioning of objectives as methodological tools to assess quality of life, there were no significant differences among patients undergoing the various procedures. However, what was evident was that all had an extremely good quality of life whichever surgical option was chosen.

Whether mucosectomy and sutured anastomosis, or stapled anastomosis should be performed is still somewhat controversial. If mucosa is left behind, what is the actual cancer risk? Another controversial area relates to whether a single versus a staged procedure should be performed and whether an ileostomy should be performed in conjunction with the pelvic pouch procedure (5).

In most instances, the stapled anastomosis leaves behind not only the transitional zone, but also 1 to $2 \mathrm{~cm}$ of anal mucosa. Lofberg et al (6) reported a case showing dysplasia and DNA aneuploidy in a pelvic pouch. This is the only reported case of dysplasia in either a pelvic pouch or a Kock pouch, and there have been no reports of cancer developing. Stern et al (1) reported cancer in an ileoanal reservoir, but the cancer originated in rectal mucosa that had been incompletely excised. This occurred in a patient who had a long rectal cuff where the indication for surgery was dysplasia. Thus, if there is severe dysplasia in the anorectal area preoperatively, options to the pelvic pouch procedure should be considered. In addition, if severe extra-intestinal manifestations of ulcerative colitis exist, then total mucosectomy - not stapled anastomosis - should be performed. There has been one other case report of cancer in a rectal cuff (6). However, with more recent techniques (leaving no rectal cuff but only the residual anal mucosa) this problem should be minimized. In addition, very few cases of anal canal cancer develop in patients who have ulcerative colitis. Therefore, in my institution, as well as in most institutions, a stapled anastomosis, leaving 1 to $2 \mathrm{~cm}$ of residual anorectal mucosa, is a safe and appropriate procedure.

We have also recently compared the outcome of patients who have had a hand-sewn ileo-anal anastomosis with that of patients who had stapled ileoanal anastomosis with or without a defunctioning ileostomy (7). Our patients were divided into three groups: group 1 included patients with a hand-sewn anastomosis and a defunctioning ileostomy, group 2 comprised patients with a stapled ileoanal anastomosis and a defunctioning ileostomy, and group 3 included patients with a stapled ileoanal anastomosis without a 
defunctioning ileostomy. The outcome measures were the leak rate, surgical complications, reoperation rate and functional outcome. Factors analyzed for their effect on the leak rate included age, sex, steroid use, weight, intraoperative difficulty, anastomotic staple technique, severity of disease at the distal margin and whether the patient had a previous subtotal colectomy.

Early surgical complications showed that the leak rate in group 1 was $12 \%, 7 \%$ in group 2 and $18 \%$ in group 3 . In group 3, 13 of 71 patients had a leak at the ileoanal anastomosis. However, only one patient in that group required a reoperation in the form of a defunctioning ileostomy. The remainder were treated with intravenous antibiotics, pouch drainage with a rectal tube and total parenteral nutrition if necessary. With this treatment regimen, 11 of the 13 patients healed their leak and were fully continent. Their functional outcome was no different than those who did not have a leak.

Seven of the 22 patients (32\%) who had a true single stage procedure developed a leak. In patients who had a previous subtotal colectomy, only $12 \%$ developed a leak. Another factor that influenced the leak rate was the amount of steroids used by patients preoperatively. As well, patients younger than 40 years had a 13\% leak rate while patients older than 40 had a $35 \%$ leak rate. In our experience, males had a $23 \%$ leak rate and females had an $11 \%$ leak rate.

It is therefore our experience and our recommendation that a true single stage procedure not be performed because of the high leak rate, but that a pelvic pouch procedure be performed without a defunctioning ileostomy in patients who have already had a previous subtotal colectomy.

\section{STRICTUROPLASTY IN THE MANAGEMENT OF CROHN'S DISEASE}

Crohn's disease management has evolved since the mid1930s from radical excision of bowel and lymph nodes to minimal surgical procedures including stricturoplasty for obstructive disease. This is justified because Crohn's disease is a panintestinal disease that appears focally and cannot be cured. Our objective, therefore, should be to alleviate symptoms. Surgery must be safe and provide minimal mortality and long term survival, but it is not absolutely necessary to remove all diseased tissue because not all of the diseased tissue will cause symptoms. It is also well known that stenosis can be overcome by either Heineke-Mikulicz or Finney type stricturoplasties. Our recent results from 1985 to 1993 following stricturoplasty in patients revealed that we performed 143 stricturoplasties in 37 patients, 25 of whom were males. The follow-up has been for a mean of 50.2 months with a range of two to 92 months. Indications for stricturoplasty in all have been obstruction. In one case, a patient with duodenal obstruction underwent stricturoplasty. The other sites of stricturoplasty included the jejunum (85 stricturoplasties), the ileum (154 stricturoplasties) and the site of previous ileocolonic anastomosis (three stricturoplasties).

In long term follow-up of these 37 patients, 20 have not been rehospitalized or reoperated upon. Three have been re- hospitalized, but not reoperated upon and 14 have been rehospitalized and reoperated upon. Of the 14 who have undergone reoperation, resection of the stricturoplasty was carried out in nine, restricturoplasty in three, a new stricturoplasty was carried out in six and resection alone in eight. The indication in all these patients for reoperation was obstruction and progression of Crohn's disease. Variables that possibly affected the reoperation rate were compared. None of the following achieved statistical significance including the type of procedure: whether the stricturoplasty was performed alone or as a resection, the type of stricturoplasty, whether the patient had undergone previous surgery, the number of stricturoplasties performed and the site of the disease. However, only two of 11 patients (18\%) who underwent stricturoplasty alone required reoperation, whereas 12 of 26 patients (46\%) who underwent stricturoplasty and resection required reoperation. Patients requiring stricturoplasty alone may represent a subset of patients who have a specific type of disease that allows a longer recurrence-free interval.

Because we have had only had one leak and one fistula in our entire series, we conclude that stricturoplasty is a safe procedure. It is effective, at least in the short term, in overcoming obstruction. Active disease may become quiescent although the natural history of the disease is usually progressive. Only patients who have multiple obstructions and who are at risk of developing a short bowel syndrome should have this procedure performed.

\section{ASSESSMENT OF TRIALS USING METHOTREXATE AND CYCLOSPORINE}

As mentioned previously, avoidance of colectomy is a poor outcome measure of medical treatment, particularly when one can cure ulcerative colitis with a surgical procedure. What appears to be lacking in a number of assessments in both medical and surgical treatments is the quality of life produced with these interventions. It is therefore very important to assess the quality of life with validated instruments, such as the time trade-off technique, direct questioning of objectives or the Inflammatory Bowel Disease Questionnaire, which has recently been described (8). It is somewhat disheartening for a surgeon to read the medical literature when statements such as "the goal of the acute phase of therapy is simply to avoid colectomy" and "methotrexate studied by Kozarek's group has already proven to be efficacious in refractory inflammatory bowel disease" appear (9). Other disheartening statements include "the much awaited controlled study from Brynskov showed effectiveness in 59\% of those patients studied with moderate Crohn's disease" (10) and that "colectomy could be avoided in the majority of patients treated with cyclosporine for severe Crohn's colitis" (11).

In assessing the results of therapy with methotrexate in Crohn's disease patients, Kozarek (12) reported on a nonrandomized, open label preliminary 12-week trial in 21 patients who had refractory IBD. Seventeen patients were on steroids. Methotrexate $25 \mathrm{mg}$ intramuscularly was given weekly 
for 12 weeks. Patients were then switched to an oral form of the drug, and were followed clinically and objectively using a modified Crohn's Disease Activity Index (CDAI) that used a score of 0 to 15 and a clinical activity index for ulcerative colitis patients that used a score of 0 to 15 . Results showed that CDAI scores fell from 13.3 to 5.4 for 11 of 14 Crohn's disease patients and that clinical activity index scores fell from 13.3 to 6.3. for five of seven ulcerative colitis patients. Although these results were encouraging, there was no determination of the dose required, the route of administration or the length of treatment.

The following year Lichtiger stated that treatment with methotrexate in this setting was efficacious (9). However, in the follow-up paper by Kozarek's group in 1992 (13), among 86 ulcerative colitis patients followed for four years, $50 \%$ had already had a colectomy and only 12 of 30 remained on methotrexate and steroids. In the Crohn's disease group, 69 patients were followed and $51 \%$ remained on methotrexate plus prednisone. In the study there was no real assessment of the quality of life and they concluded that a significant number of patients with severe inflammatory bowel disease (IBD) became refractory to methotrexate. Although their early results might have been encouraging, the later followup showed a refractoriness to the drug, as well as the potential for long term side effects while using this drug. One must also consider the ethical dilemma of treating ulcerative colitis patients with long term immunosuppressive drugs when ulcerative colitis is a potentially curable disease when treated surgically.

Aurora et al (14) reported on a double-blind, placebo controlled trial of methotrexate in Crohn's disease, and although there were fewer flare-ups on methotrexate, side effects were very significant and compliance was poor, with only $31 \%$ of patients on methotrexate completing a 52 -week course of the drug.

Lichtiger et al (9) reported in 1990 on 24 ulcerative colitis patients who underwent both an acute and chronic phase of cyclosporine therapy. Nineteen of 24 patients were considered acute responders and 13 of 19 were considered chronic responders. Overall, the follow-up was short and only 13 of 24 succeeded. These patients were followed using a modified clinical activity index; a score of greater than 12 determined severe disease, 6 or less indicated success in the acute phase (a score reduction of at least $50 \%$ also denoted success in the acute phase) and 4 or less represented a successful outcome in the chronic phase of their study. Once again, no quality of life assessments were undertaken in this study. The disease severity assessment was weighted with the following outcome measures: diarrhea, nocturnal diarrhea, blood in the stool, fecal incontinence, abdominal pain and cramping, general well-being, abdominal tenderness and antidiarrheals.

In the acute phase of this study - wherein a score of 6 or less or a $50 \%$ reduction in the severity of the clinical index indicated success - one can use examples to show that patients who had seven to nine bowel movements per day with some blood in the stool, abdominal cramping and localized tenderness can have a score of 6 . As far as a surgeon is concerned, localized tenderness can be a very serious problem and one must question whether a patient with a clinical activity of 6 manifesting the symptoms mentioned above should be considered a success. In addition, a patient who feels poorly having five to six bowel movements per day could also have a score of 6 . In the chronic phase, where success was related to a score of equal to or less than 4 , a patient who had only an average general well-being with localized tenderness could also have a score of 4 . In addition, a patient with severe or rebound tenderness with three to four bowel movements per day could have a score of 4 . Of interest is the fact that six of the first 15 patients entered into the chronic phase actually had a clinical activity score of greater than 6 (average 7 to 9 ).

In a follow-up study reported in 1993 using intravenous cyclosporine $4 \mathrm{mg} / \mathrm{kg} /$ day and switching to oral cyclosporine $8 \mathrm{mg} / \mathrm{kg} /$ day when a successful outcome was achieved, nine of 11 patients were successful in the acute phase on intravenous cyclosporine and none of the nine on placebo were considered successful until some were crossed over to receive intravenous cyclosporine (15). There appeared to be an advantage to using intravenous cyclosporine in acute phase therapy of ulcerative colitis. What are the potential long term side effects using cyclosporine and what is the refractoriness of the disease to cyclosporine? What is the potential for malignancy if one were to maintain a patient on cyclosporine and steroids? These are very important questions to ask before suggesting cyclosporine use for these patients.

As far as cyclosporine use in Crohn's disease is concerned, the study by Brynskov et al (16) showed that $59 \%$ of patients on cyclosporine over a three-month period improved, but so $\operatorname{did} 32 \%$ of the placebo patients. In addition, $36 \%$ of the patients relapsed after stopping cyclosporine treatment, a result that again brings up the question of how to treat the patient in the long term. The Canadian Crohn's Relapse Prevention Trial showed no improvement in Crohn's patients treated with cyclosporine and no improvement in the CDAI, quality of life or percentage of steroids used (17). In the low activity stratum, the placebo group did better than the cyclosporinetreated group. In the study reported by Leddin and colleagues (11), avoidance of colectomy again was used as an outcome measure. Twenty-one per cent had early colectomy, another $21 \%$ came to colectomy an average of 73 weeks after initiation of therapy and $21 \%$ had stricturoplasty or small bowel resection but did not have their colons removed. Therefore, in assessing this study, only $27 \%$ of patients did not come to surgery. Of importance was that there were three deaths, two related to sepsis and one to a cerebrovascular accident. All these deaths could be attributed to cyclosporine use. Although the conclusion from the study was that colectomy could be avoided in the majority of patients treated with cyclosporine for severe colitis, a surgical viewpoint would examine this with extreme scepticism and ask at what price is this avoidance of colectomy or surgery. 


\section{TOXICITY OF MEDICATIONS AND SURGERY}

In addition to looking strictly at outcome measures, one must consider the potential toxic effects of medical treatment and the potential morbidity incurred with surgery. The mainstay of therapy for patients with Crohn's disease and a number of patients with ulcerative colitis is steroids. However, side effects of using long term corticosteroids are significant and include moon facies in $47 \%$ of patients, acne in $30 \%$, infection in $27 \%$, striae in $23 \%$, depression in $20 \%$, hypertension in $13 \%$, osteopenia in $30 \%$, osteonecrosis in $4 \%$ and psychosis in $2 \%$, and growth retardation, pancreatitis and diabetes in a number of patients.

Cyclosporine also has a great potential for producing side effects. In reviewing the literature on the use of cyclosporine in IBD, major toxic effects include nephrotoxicity (in 7.5\% to $10 \%$ of patients), paresthesia (36 to $45 \%$ ), hypertension (20\%), headache $(5 \%)$, seizures and other neurological events ( $9 \%$ to $12 \%)$, hypertrichosis ( $25 \%)$, gastrointestinal distress (10\%) and gingival hypertrophy (5\%) (18). In addition, it must be emphasized once again that the combination of cyclosporine and steroids may produce malignancy which heretofore has not been reported.

Methotrexate toxicity includes pneumonitis, gastrointestinal distress and biochemical liver abnormalities (14). Although medical therapy is associated with very significant toxic effects, one must also consider the complications of ileostomies and pelvic pouch surgery in ulcerative colitis patients undergoing these procedures. The sepsis, leak, abscess and fistula rates following the pelvic pouch procedure range from $8 \%$ to $15 \%$. Although not specific for the operation, intestinal obstruction occurs in $10 \%$ to $20 \%$ of cases, pouchitis from $10 \%$ to $30 \%$, sexual dysfunction in both males and females in $5 \%$ to $10 \%$, strictures in 5\%, loop ileostomy complications in $25 \%$ and pouch excision in $5 \%$ to $8 \%(19,20)$.

\section{CONCLUSIONS}

Both medical and surgical therapies have their own resultant toxicity and morbidity and they must be taken into consideration when managing these patients. It is important to think of an IBD patient undergoing a combined multidisciplinary approach from both the physician and the surgeon. It is no longer acceptable for one or the other group, each with its own biases, to treat these patients in isolation. To that end, it is my view that both medical and surgical and/or combined trials of various medications or surgery should be undertaken. Importantly, before using long term immunosuppressive agents, one must consider the quality of life produced while being on medical agents versus surgical therapy.

For ulcerative colitis patients, therefore, it is my strong belief that long term management with potent immunosuppressives is potentially dangerous in a surgically curable disease. The results of surgery are certainly not perfect, but the quality of life will be improved with whatever surgical procedure is undertaken. Avoidance of colectomy is a poor indicator of the results without factoring in quality of life measurements, and the perception of surgery or a stoma is far worse than reality for most patients.

In Crohn's disease, both medical and surgical therapies have a role. Methotrexate and cyclosporine do not appear to be the long term answer for these patients. As far as stricturoplasty is concerned, although it is effective in overcoming obstructive episodes, it does not appear to change the natural history of the disease in the long term.

Management of patients with IBD can be extremely challenging and difficult. Patients, however, must be well aware of all therapy options - both medical and surgical - and must be involved in the decision-making process. Gastroenterologists and surgeons must come together in their thinking about IBD patients and continue to undertake trials of both medical and surgical therapies in order to determine the best management for any individual patient.

\section{REFERENCES}

1. Stern H, Walfisch S, Mullen B, McLeod RS, Cohen Z. Cancer in an ileo-anal reservoir - A new late complication. Gut 1990;3:473-5.

2. Fleshman JW, Cohen Z, McLeod RS, Stern H, Blair J. The ileal reservoir and ileo-anal anastomosis procedure - Factors affecting technical and functional outcome. Dis Colon Rectum 1989;31:10-6.

3. McIntyre TB, Pemberton J, Wolff B, Beart R, Dozois R. Comparing functional results one year and ten years after ileal pouch anal anastomosis for chronic ulcerative colitis. Dis Colon Rectum 1994;37:303-7.

4. McLeod RS, Churchill N, Lock A, Vandenburg H, Cohen Z. Assessment of quality of life of patients with ulcerative colitis preoperatively and postoperatively. Gastroenterology 1991;101:1307-13.

5. Cohen Z. Ileo-anal pouches - Is mucosectomy necessary? Can J Gastroenterol 1993; 7:1-3.

6. Lofberg R, Lindquist L, Lindquist K, Veress B, Reinholt FP, Tribukait B. Dysplasia \& DNA aneuploidy in a pelvic pouch. Dis Colon Rectum 1991;34:280-4.

7. Cohen Z, Steven W, O'Connor B, Stern H, McLeod RS. Continuing evolution of the pelvic pouch procedure. Surgery 1992;216:506-12.

8. Archambault A, Feagan B, Fedorak R, et al. The Canadian Crohn's Disease Relapse Prevention Trial (CCRPT). Gastroenterology 1992;102:A591.

9. Lichtiger S. Cyclosporine therapy in inflammatory bowel disease Open-label experience. Mt Sinai J Med 1990;57:315-9.

10. Lichtiger S, Present DH. Preliminary report: Cyclosporine in treatment of severe active ulcerative colitis. Lancet 1990;336:16-9.

11. Leddin DJ, Vanzanten V, Sidorov JJ, Stewart J, Tripathi D, Williams CD. Colectomy following treatment of Crohn's colitis with cyclosporin. Poster Presentation. Trends in Inflammatory Bowel Disease Therapy, Victoria, April 1994.

12. Kozarek RA, Patterson DJ, Gelfand MD, Botoman VA, Ball TJ, Wilske KR. Methotrexate induces clinical and histologic remission in patients with refractory inflammatory bowel disease. Ann Intern Med 1989;110:353-6

13. Kozarek RA, Patterson DJ, Gelfand MD, Ball TJ, Botomon VA. Long-term use of methotrexate in inflammatory bowel disease: Severe disease 3, Drug therapy 2. Seventh inning stretch. Gastroenterology 1992;102:A648. (Abst)

14. Arora S, Katkov WN, Cooley J, et al. A double blind, randomized, placebo-controlled trial of methotrexate in Crohn's disease. Gastroenterology 1993;102:A647.

15. Lichtiger S, Present DH, Kornbluth A, Hanauer S. Cyclosporin A in the treatment of severe, refractory ulcerative colitis: A double blinded placebo controlled trial. Gastroenterology 1993;102:A732.

16. Brynskov J, Freund L, Norby Rasumussen S, et al. Final report on a placebo-controlled, double-blind, randomized, multicentre trial of cyclosporin treatment in active chronic Crohn's disease. Scand J Gastroenterol 1991;26:689-95.

17. Archambault A, Feagan B, Fedorak R, et al. The Canadian Crohn's Relapse Prevention Trial. Gastroenterology 1992;4:A591. 


\section{Cohen}

18. Corman E, Reinus JF. Cyclosporine use in steroid-resistant Crohn's disease - Grasping at new straws? Am J Gastroenterol 1990;85:758-9

19. Pemberton JH, Kelly KA, Beart RW, Dozois R, Wolff B, Ilstrup DM.

Ileal pouch anal anastomosis for chronic ulcerative colitis - Long term results. Ann Surg 1987;206:504-13.
20. Salemans JM, Nagengast M, Cubbers EJ, Kuijpers JH. Postoperative and long-term results of ileal pouch anal anastomosis for ulcerative colitis in familial polyposis. Dig Dis Sci 1992;37:1882-9. 


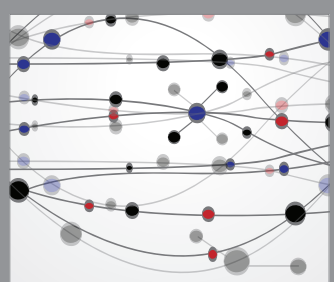

The Scientific World Journal
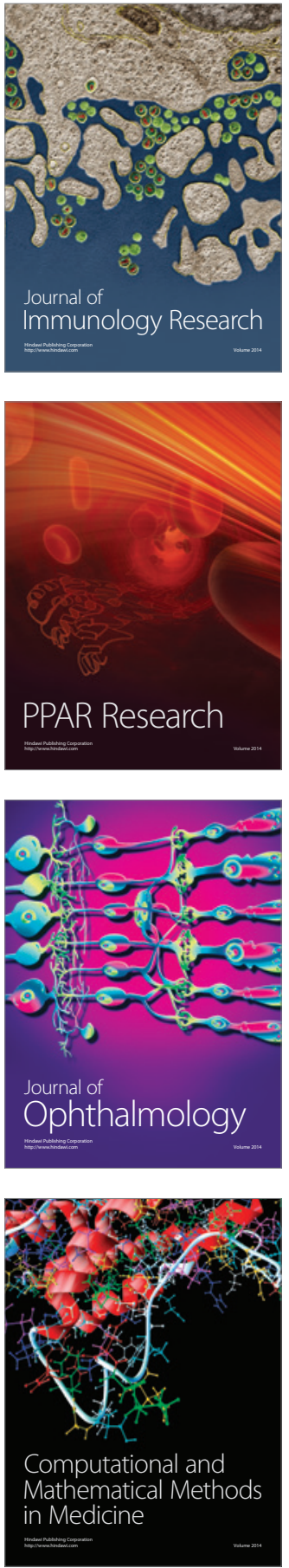

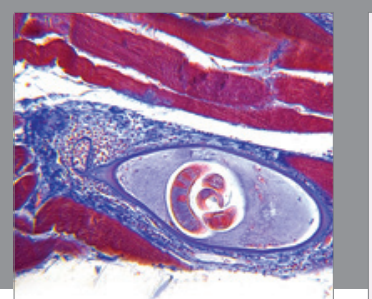

Gastroenterology Research and Practice

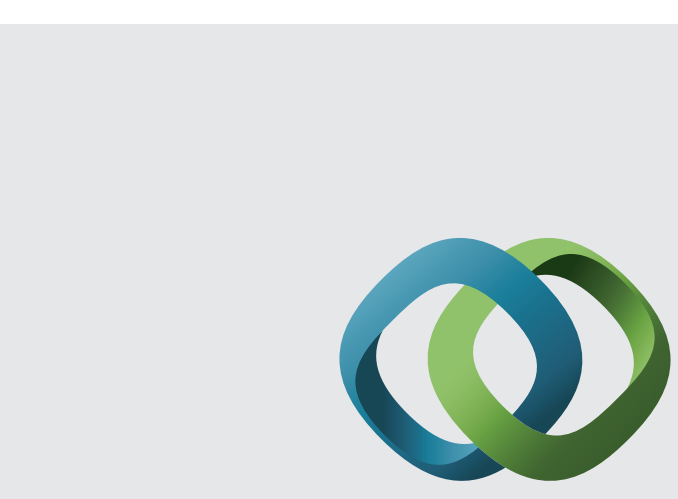

\section{Hindawi}

Submit your manuscripts at

http://www.hindawi.com
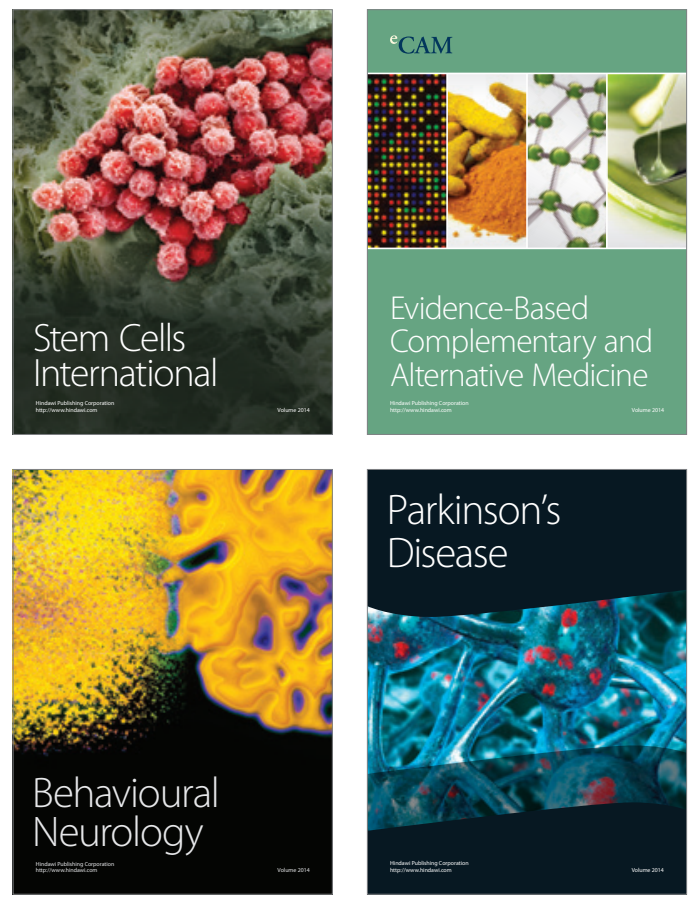
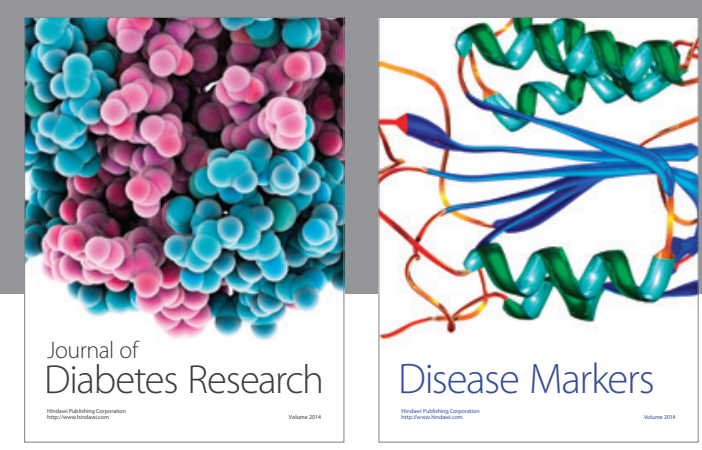

Disease Markers
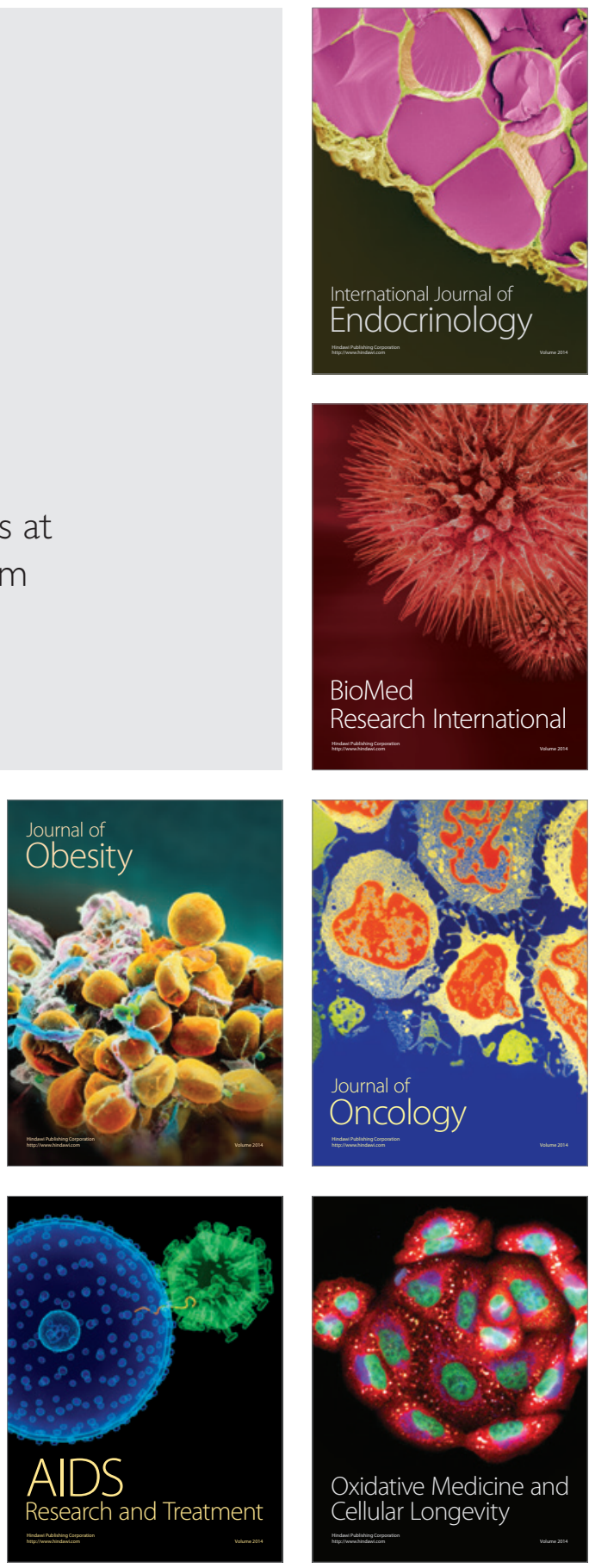